\title{
THE
}

\section{Complementary and Alternative Medicine Use and Adherence to Asthma Medications among Latino and Non-Latino White Families}

Elizabeth L. McQuaid

David A. Fedele

Sue K. Adams

University of Rhode Island, suekadams@uri.edu

Daphne Koinis-Mitchell

5oldosytthisiand_afpditional works at: https://digitalcommons.uri.edu/hdf_facpubs

The University of Rhode Island Faculty have made this article openly available.

Please let us know how Open Access to this research benefits you.

This is a pre-publication author manuscript of the final, published article.

Terms of Use

This article is made available under the terms and conditions applicable towards Open Access Policy Articles, as set forth in our Terms of Use.

\section{Citation/Publisher Attribution}

McQuaid E.L., Fedele, D.A., Adams, S.K., Koinis-Mitchell, D., Mitchell, J.M., Kopel, S.J., Seifer, R., Jandasek, B., Fritz, G.K., \& Canino, G. (2014). Complementary and alternative medicine use and adherence to asthma medications among Latino and non-Latino white families. Academic Pediatric, 14(2),192-199. doi: 10.1016/j.acap.2013.09.006

Available at: http://dx.doi.org/10.1016/j.acap.2013.09.006

This Article is brought to you for free and open access by the Human Development and Family Science at DigitalCommons@URI. It has been accepted for inclusion in Human Development and Family Science Faculty Publications by an authorized administrator of DigitalCommons@URI. For more information, please contact digitalcommons-group@uri.edu. 


\section{Authors}

Elizabeth L. McQuaid, David A. Fedele, Sue K. Adams, Daphne Koinis-Mitchell, Jessica Mithcell, Sheryl J. Kopel, Ronald Seifer, Barbara Jandasek, Gregory K. Fritz, and Glorisa Canino 


\title{
Complementary and Alternative Medicine Use and Adherence to Asthma Medications among Latino and Non-Latino White Families
}

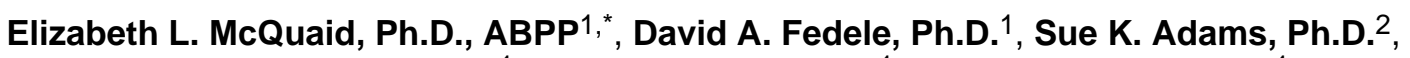 \\ Daphne Koinis-Mitchell, Ph.D. ${ }^{1}$, Jessica Mitchell, M.S ${ }^{1}$, Sheryl J. Kopel, M.Sc. ${ }^{1}$, Ronald \\ Seifer, Ph.D. ${ }^{1}$, Barbara Jandasek, Ph.D. ${ }^{1}$, Gregory K. Fritz, M.D. ${ }^{1}$, and Glorisa Canino, Ph.D. \\ 3 \\ ${ }^{1}$ Bradley/Hasbro Children's Research Center: Alpert Medical School of Brown University, \\ Providence, RI \\ ${ }^{2}$ Department of Human Development and Family Studies, University of Rhode Island, Kingston, \\ RI \\ ${ }^{3}$ Behavioral Sciences Research Institute, University of Puerto Rico Medical Sciences Campus, \\ San Juan, Puerto Rico
}

\begin{abstract}
Objective-The current study sought to evaluate patterns of complementary and alternative medicine (CAM) use in a sample of Latino and Non-Latino white (NLW) children with asthma, to determine whether parental beliefs about conventional medications and barriers to obtaining these medications were related to CAM use, and to assess whether CAM use was associated with decreased adherence to controller medications.
\end{abstract}

Methods-Participants included 574 families of children with asthma from Non-Latino White, Puerto Rican, and Dominican backgrounds from RI and from Island Puerto Rico. All parents completed a brief checklist of barriers to medication use and an assessment of CAM approaches. A subsample of 259 families had controller medication use monitored objectively for approximately one month by MDILog (fluticasone propionate), TrackCap (montelukast), or dosage counter (fluticasone/salmeterol combination).

Results-Prevalence of CAM use was high among Latino families. Perceived barriers to obtaining medication were related to increased CAM use in Puerto Rican families from RI. Elevated medication concerns were positively associated with CAM use among NLW and Island PR families. CAM use was positively related to objective adherence within NLW families, and unrelated in other groups.

Conclusions-CAM use is common among Latino families with asthma. Among some families, CAM use may be initiated as a way to cope with barriers to obtaining medication or when parents

(C) 2013 Academic pediatric Association. Published by Elsevier Inc. All rights reserved.

*Elizabeth L. McQuaid, Ph.D., ABPP, Bradley/Hasbro Children's Research Center, 1 Hoppin St. Providence, RI 02903, Elizabeth_McQuaid@brown.edu, Phone: 401-444-7573, Fax: 401-444-5143.

Conflict of Interest: The authors have no relevant conflicts of interest to declare.

Publisher's Disclaimer: This is a PDF file of an unedited manuscript that has been accepted for publication. As a service to our customers we are providing this early version of the manuscript. The manuscript will undergo copyediting, typesetting, and review of the resulting proof before it is published in its final citable form. Please note that during the production process errors may be discovered which could affect the content, and all legal disclaimers that apply to the journal pertain. 
have concerns about conventional medications. Families who report CAM use do not appear to be substituting CAM for conventional asthma medication.

\section{Keywords}

Asthma; Adherence; Complementary and Alternative Medicine

Pediatric asthma is associated with impaired quality of life and increased health care utilization (1). Minority and disadvantaged populations are disproportionately affected (2). Latinos of Caribbean have particularly high prevalence, morbidity, and health care utilization (3). The sources of these disparities are multifaceted and complex, and include genetics, individual and family beliefs, health care system features, and broad environmental variables such as housing and pollution (4). Attitudes toward conventional medication use, and barriers to obtaining prescribed medications, may be modifiable sources of asthma disparities (5).

National guidelines for asthma management include daily controller medication use to prevent symptoms and exacerbations for those with chronic asthma (6). Research among ethnic minorities consistently demonstrates concerns about controller medication use $(7 ; 8)$, and lower medication adherence (9). We previously found that controller medication adherence was low among island Puerto Rican and Latino families in the United States relative to Non-Latino white families (10). Data indicate greater acceptance of complementary and alternative medicine (CAM) use among ethnic minority families with asthma (11). Few studies, however, have examined how preference for CAM may relate to adherence to prescribed controller medications (12).

CAM is defined as "a group of diverse medical and health care systems, practices, and products that are not generally considered part of conventional medicine" (13). Prevalence of CAM use among families of children with asthma ranges from 33-89\% across studies (14). Rates of CAM use for ethnic minority families with asthma range between 48-89\%, depending upon ethnicity (15-16). Reasons for CAM use in ethnic minority families are likely multifaceted. In earlier work examining CAM use among Puerto Rican families, patterns of acculturation were related to medication adherence, with higher adherence among families whose parents reported a bicultural orientation (16). CAM use may be related to families' broader ethnocultural belief systems regarding health and illness (17).

\section{Potential Relation of CAM Use to Beliefs and Barriers}

Parental beliefs and concerns about conventional asthma medication may be associated with CAM use. Studies including Latino $(5 ; 8)$ and mixed ethnicity samples $(7 ; 18)$ indicate that parents often have concerns surrounding the use of conventional asthma medication, including immediate side-effects, medication dependence, and long-term effects. These concerns are related to lower reported asthma medication adherence (7) and higher likelihood of uncontrolled asthma (19). Studies relating parental medication beliefs and child asthma medication adherence, however, have used predominantly NLW samples and have not assessed CAM use (18).

Ethnic minority families may be more likely to face barriers to obtaining prescription asthma medications (20). These include financial burden, socio-cultural factors (e.g., limited English proficiency), and practical constraints (e.g., transportation difficulties) (21). For instance, CAM use has been found to be higher among uninsured families and those who perceived financial barriers to asthma care (22). In one report from the sample presented in this paper, barriers to medication use were high among Island PR families relative to Latino 
and non-Latino white families (5). These barriers also may play a role in greater CAM use and lower adherence to conventional asthma medications.

\section{CAM and Adherence to Asthma Medication}

Latino parents of children with asthma may substitute CAM for asthma medications to prevent symptoms (8). CAM may also be used as a first line treatment for episodes (15). Research regarding CAM use and adherence to controller medications; however, has produced mixed findings. Positive caregiver beliefs surrounding CAM were linked to risk for self-reported nonadherence to asthma medication in a largely minority sample (23). Alternatively, parent report of child medication adherence was not associated with initiation of CAM within a predominantly NLW sample (12). To date, no studies have examined whether CAM use is associated with asthma medication adherence using objective electronic monitoring methods, and whether that association differs by ethnic group.

\section{The Current Study}

In a prior report including this sample, we found that adherence to controller medications differed by ethnic group, with the lowest adherence among families of children from Island Puerto Rico, and the highest among NLW families (10). Rates of CAM use; however, and the role of CAM in relation to medication adherence have not yet been reported. In the current study, we evaluated CAM use in a sample of families of children with asthma from NLW, Puerto Rican, and Dominican backgrounds from RI and Island PR, to evaluate patterns of overall CAM use by ethnic group. We also sought to determine whether parental beliefs about conventional asthma medications and barriers to obtaining medications were related to CAM use, and if language preference and years living outside the US (proxies for acculturation) were associated with CAM use among our mainland Latino subgroups (Puerto Rican and Dominican). Lastly, we evaluated whether CAM use was associated with objective adherence to asthma controller medications. We hypothesized that: 1) CAM use would be higher in families of Puerto Rican and Dominican descent, relative to NLW families, 2) negative beliefs surrounding conventional asthma medication and barriers to asthma medication use would be related to greater CAM use for all families, 3) lower levels of acculturation would be associated with reports of CAM use for mainland Latino families, and 4) increased CAM use would be related to lower levels of adherence to controller medications, but only among Latino families $(12 ; 23)$.

\section{Method}

Participants were a subset from a larger study $(N=805)(24)$ evaluating mechanisms underlying pediatric asthma disparities between Latino and NLW groups. Previous published work from this study has addressed contributing factors to variations in asthma outcomes across Latino and NLW children, such as asthma severity (25), asthma symptom perception (26), and objective rates of controller medication use (10).

Data collection occurred in Puerto Rico (PR) and Rhode Island (RI). Participants (aged 7-16 years) were recruited from hospital-based and community primary care clinics, community events, and asthma classes. Asthma diagnosis was confirmed through medical history, physical examination, and spirometry; those with complicating respiratory conditions were excluded. Puerto Rican families with a child with asthma were eligible at the PR site. Ethnicity in RI was determined by parent report, and caregivers of Puerto Rican, Dominican, or NLW ethnicity were eligible. For this report, we include only children with persistent asthma (due to greater medication need), and those that completed an evaluation of CAM use. 


\section{Procedure}

Data collection occurred in four study visits over the course of approximately four months. Visits were generally conducted in the home by research assistants; the clinic visit was conducted in a hospital setting by a study physician or nurse practitioner. Informed consent and demographic information were obtained at enrollment. Data included in this study were collected at the second visit, typically $1-2$ weeks after enrollment. During this visit, the family was interviewed regarding asthma management strategies using a standardized interview (27), completed surveys, and was oriented to adherence monitoring procedures. Adherence devices were collected approximately five weeks later. Families were compensated for participation. The protocol was approved by institutional review boards at both sites.

\section{Measures}

Asthma Severity-Asthma severity was evaluated by a study clinician during the clinic visit, based on physical exam, history, report of symptom frequency, pre-albuterol FEV1, and current controller medication dose. The four levels of asthma severity were "Mild Intermittent", "Mild Persistent", "Moderate Persistent", and "Severe Persistent", consistent with guidelines in place at the time of the study (28).

Medication Beliefs-Parents completed a ten-item self-report measure (29) to assess parental beliefs about asthma medication, resulting in index scores of perceived medication necessity (e.g., the medicines are necessary for the child's health) and medication concerns (e.g., dependence, side effects).

Barriers to Filling Prescriptions-Potential barriers to filling prescriptions were evaluated using an 8 -item list devised for the larger study $(20 ; 23)$. Items represented health care system and contextual barriers such as cost, transportation problems, language barriers, and pharmacy wait-time.

Acculturation-Language preference was coded as preferred language of research interview for RI Puerto Rican and Dominican families; this categorical variable and the number of years the caregiver had lived outside of the mainland US were used as proxies for acculturation.

CAM Checklist-CAM use was assessed with the CAM Checklist, developed for the larger study to assess CAM use among Latino and NLWs (23), administered in the context of an established asthma management interview (27). Items were originally derived through literature review (17), ongoing research with the target population (30) and a series of focus groups with families from different ethnic subgroups. A list of asthma treatment strategies reported by families was independently reviewed by experts in asthma management and cultural issues in RI and PR. Participants indicated whether or not they had utilized various types of CAM to treat their children's asthma.

Medication Adherence Monitoring-A subset of participants who were prescribed asthma controller medications that could be monitored objectively participated in the medication adherence monitoring protocol. Objective adherence monitoring in the larger study is described in detail elsewhere (10). In brief, adherence was monitored for controller medications in three distinct ways. Adherence to oral montelukast was monitored using the TrackCap MEMS monitor (AARDEX), which is a replacement pill bottle with an electronic cap that registers the date and time of each opening. Adherence to inhaled fluticasone was monitored using The MDIlog II (Westmed), a small electronic device attached to an inhaler "sleeve" that fits over the inhaler, and records the date and time of inhaler actuation with 
inhalation. Adherence to fluticasone/salmeterol combination medication (diskus) was monitored by using a standard protocol to record dosage counter readings at the beginning and end of the monitoring period.

Adherence was monitored for a minimum of 28 days. Data were inspected and cleaned according to standard procedures (31). Seventy-five cases were eliminated from the adherence analysis for a range of reasons (see (10) for specific details regarding device loss and data cleaning), resulting in 277 usable cases. Of these, 259 families had also completed the CAM Checklist. Data used in adherence analyses were available from 77 island PR children, 51 RI PR children, 56 RI DR children, and 75 RI NLW children.

Adherence estimates were calculated as total doses per day/prescribed doses per day. For participants on more than one monitored medication, the adherence estimate represented the average of the two adherence measurements. Given that participants could be prescribed more than one monitored medication, and medication adherence is known to differ by medication type $(32 ; 33)$, a categorical variable was constructed to represent each medication combination encountered in this study, namely montelukast alone (61.4\%), fluticasone alone (7.7\%), fluticasone/salmeterol combination (8.9\%), fluticasone and montelukast (8.5\%), and fluticasone/salmeterol combination with montelukast (13.5\%).

\section{Overview of Analyses}

Chi square analyses were conducted to determine whether the prevalence of CAM use and CAM types differed by site/ethnicity. T tests, chi square analyses, and logistic regressions were conducted to examine whether acculturation variables, caregiver perceived barriers to prescription medication, and beliefs about asthma medication were predictive of CAM use (yes/no). The total number of barriers to prescription medication and the medication necessity and concerns subscales were entered simultaneously in each regression model.

Adherence estimates, which represent proportional data, were adjusted using a probit transformation prior to analysis. Regression analyses controlling for covariates, specifically the child's asthma severity level, medication monitoring type (e.g., MDIlog, TrackCap, or dosage counter; 32), and child age (31) were conducted to determine whether reported CAM use was associated with objective medication adherence.

\section{Results}

\section{Participants}

From an initial sample of 805 families, the current sample included children with persistent asthma who completed the CAM checklist. This resulted in a sample of 574 families across sites, including 267 island PR children, 92 RI PR children, 107 RI DR children, and 108 NLW children in RI (Table 1). A subsample of 259 children also had objective adherence data available (see Figure 1).

\section{Prevalence of overall CAM use by Site/Ethnicity}

Reported CAM use differed by site and ethnicity $\left(\chi^{2}(3, N=574)=97.01, p<.001\right)$. Island PR families reported the highest rates of CAM use (73\%) compared to RI PR (51\%), RI DR (62\%), and NLW families (19\%). Subsequent analyses were conducted within groups.

\section{Prevalence of CAM Type across Site/Ethnicity}

Reported types of CAM also differed by site and ethnicity; specifically, mind-body interventions $\left(\chi^{2}(3, N=574)=81.70, p<.001\right)$, biologically-based therapies $\left(\chi^{2}(3, N=\right.$ $574)=91.63, p<.001)$, and body-based methods $\left(\chi^{2}(3, N=574)=101.30, p<.001\right)$ all 
differed by our site/ethnicity groups (see Figure 2). Island PR families reported the highest rates of CAM use across the three types, followed by RI DR, RI PR, and NLW families. Data regarding use by CAM item and type are presented in Table 2.

\section{Association of CAM Use to Acculturation Variables, Perceived Barriers, and Medication Beliefs}

Acculturation variables-The number of years caregivers reported living outside of the US was unrelated to CAM use across groups. Language preference was only related to CAM use in RI DR families, $\chi^{2}(1, N=125)=5.34, p=.021$. CAM use was higher among Spanish speaking (57.6\%) than English speaking (5.6\%) RI DR families.

Perceived barriers to prescription medication-A higher number of perceived barriers to prescription medication use was associated with CAM use among RI PR families, $\beta=.76, S E=.39, p=.050$ (Table 3), but not among other groups.

Beliefs about medication-Elevated medication concerns were associated with CAM use among Island PR $(\beta=.50, S, .22, p E=.026)$ and RI NLW $(\beta=.85, S, .39, p E=.030$; see Table 3) families, but not among RI PR and RI DR families. Medication necessity was not related to CAM use within any group.

\section{Relation of CAM to adherence}

CAM use was related to increased adherence to controller medications within NLW families, $\beta=.23, p=.047$ (Table 4). For children and families from Latino backgrounds, CAM use was not related to controller medication adherence.

\section{Discussion}

Pediatric asthma continues to represent a source of health disparities between Latinos and NLWs $(1 ; 3)$. Given that adherence to controller medications remains challenging for many families $(31 ; 34)$, and for minority families in particular $(10 ; 32 ; 33)$, cognitive and behavioral variables such as attitudes toward medication use, barriers to obtaining medications, and preference for CAM use represent potentially modifiable intervention targets. In our sample, nearly three quarters of Island PR and over half of RI Latino families reported some CAM use, whereas only one fifth of NLW families reported using CAM. Results are consistent with previous research demonstrating a high prevalence of CAM use in Latino families $(17,30)$.

Self-report of CAM use was associated with language preference among our Dominican group, suggesting that lower-acculturated families were more likely to use CAM. CAM use was modestly associated with beliefs and barriers to medication use, and only for certain groups. Among our RI PR group, barriers to obtaining medications were modestly associated with CAM use. It is possible that these families may turn to CAM when they encounter barriers to obtaining asthma medication. Due to financial strain, barriers to obtaining medications may also serve as barriers to obtaining CAM (e.g., rubs available at the pharmacy, teas). Elevated medication concern was positively associated with CAM use only in the Island PR and NLW groups. Parental concerns could be a factor in the high prevalence rate of CAM use within the Island PR group, and similar concerns may play a role among NLW families. Specific factors that may predict CAM use not measured in this report bear further study, including ease of access to culturally sanctioned CAM methods, such as through a bodega or herbalist in one's neighborhood. A more nuanced examination of acculturation and immigration factors (e.g., cultural identity), health-care system factors 
(e.g., consistent asthma care provider), and CAM use in relation to conventional medication use is warranted.

We previously noted the lowest rates of adherence to controller medications among Island PR families, and the highest rates among RI NLW families (10). Our results suggest that factors other than CAM use may account for this effect. CAM use was unrelated to medication adherence among our Latino families in both RI and PR. These findings are consistent with previous research (12), indicating no relation between child medication adherence and CAM use in a predominantly NLW sample.

CAM use was positively related to medication adherence among NLW families in our sample. Some families who are concerned about their child's asthma may try multiple strategies (both conventional and CAM) to manage symptoms. For Latino families in RI and PR this relationship is less clear. CAM use may be related to medication use at multiple levels, and may change over time due to family circumstances. For example, families may hesitate to fill a prescription once received, or use it intermittently according to their financial circumstances. If a medication has run out and they do not have the resources for a refill, praying or using oils may seem a viable alternative if useful in the past. Overall, these results reflect that the relationship between CAM use and use of conventional medications is complex, and CAM use may not indicate poor medication adherence. Research is needed to clarify dynamic, multi-level factors that may predict CAM use and use of conventional asthma medications among specific ethnic groups.

A number of study limitations bear mention. Our checklist was developed to assess CAM use specific to Latinos, and may have missed certain strategies more typically implemented by NLW families (e.g., chiropractic care; 35). Use of objective measures of medication adherence is a strength; however, we were only able to assess adherence to medications that had been prescribed and filled, and hence did not capture under prescription of controller medications, or nonadherence through failure to fill prescriptions. Qualitative work may be necessary to fully understand how families make decisions about CAM use, and how this affects patterns of filling and using conventional medications.

Although study personnel were fully bilingual and trained in cultural sensitivity surrounding asthma practices, it is possible that RI Latino families underreported CAM use due to perceptions that these strategies might be less acceptable in the dominant US culture. Additionally, administration of measures regarding asthma management prior to adherence data collection may have "primed" families to be more adherent; however, it is not clear this would lead to group differences. Group sample sizes were modest, which may have limited our power to find effects. Lastly, our sample was recruited based on likely asthma diagnosis and through convenience sampling, and hence may not adequately represent families with children under diagnosed or undertreated for asthma.

There is an increasing emphasis on culturally-centered care in the treatment of children with asthma (36). Incorporating the cultural beliefs and practices of families into asthma-related care can improve asthma outcomes compared to standard treatment approaches (37). Following the Awareness-Assessment-Negotiation model (38) may aid clinicians in integrating these findings into patient care. According to this model, clinicians should become aware of commonly held beliefs and practices among cultures for which they provide care, assess these beliefs and practices, and find ways to integrate beliefs and practices into patient care. Clinicians should be encouraged to discuss and assess CAM use and how it may be related to concerns regarding use of conventional asthma medications and family-specific barriers to medication use (17). Culturally-centered care may enhance 
the patient-provider relationship and ultimately improve asthma management among families of children at most risk for asthma morbidity.

\section{Acknowledgments}

Funding: This work was funded by the National Heart, Lung, and Blood Institute; U01 HL 072438, G. Fritz and G. Canino, PIs, and Eunice Kennedy Shriver Institute of Children's Health and Human Development, K24 HD 058794, E.L. McQuaid, PI. Funded by the National Institutes of Health (NIH).

\section{References}

1. Akinbami LJ, Moorman JE, Liu X. Asthma prevalence, health care use, and mortality: United States, 2005-2009. Natl Health Stat Report. 2011; (32):1-14. [PubMed: 21355352]

2. Hunninghake GM, Weiss ST, Celedón JC. State of the art: Asthma in Hispanics. American Journal of Respiratory and Critical Care Medicine. 2006; 173(2):143-163. [PubMed: 16210666]

3. Lara M, et al. Heterogeneity of childhood asthma among Hispanic children: Puerto Rican children bear a disproportionate burden. Pediatrics. 2006; 117:43-53. [PubMed: 16396859]

4. Canino G, McQuaid EL, Rand CS. Addressing asthma health disparities: A multilevel challenge. Journal of Allergy and Clinical Immunology. 2009; 123(6):1209-1217. [PubMed: 19447484]

5. McQuaid EL, et al. Beliefs and barriers to medication use in parents of Latino children with asthma. Pediatric Pulmonology. 2009; 44(9):892-898. [PubMed: 19672958]

6. National Asthma Education and Prevention Program . Expert Panel Report 3: guidelines for the diagnosis and management of asthma. US Department of Health and Human Services, National Institutes of Health; Bethesda MD: 2007.

7. Conn KM, et al. Parental beliefs about medications and medication adherence among urban children with asthma. Ambulatory Pediatrics. 2005; 5(5):306-10. [PubMed: 16167856]

8. Bearison DJ, Minian N, Granowetter L. Medical management of asthma and folk medicine in a Hispanic community. Journal of Pediatric Psychology. 2002; 27(4):385-392. [PubMed: 11986361]

9. Apter A, et al. Adherence with twice-daily dosing of inhaled steroids. Socioeconomic and healthbelief differences. American Journal of Respiratory and Critical Care Medicine. 1998; 157(6 Pt 1): 1810-7. [PubMed: 9620910]

10. McQuaid EL, et al. Medication adherence among Latino and non-Latino white children with asthma. Pediatrics. 2012; 129(6):e1404-10. [PubMed: 22566417]

11. Adams S, Koinis Mitchell D. Perspectives on complementary and alternative therapies in asthma. Expert Rev Clin Immunology. 2008; 4(6):703-711. [PubMed: 20477120]

12. Philp JC, et al. Complementary and alternative medicine use and adherence with pediatric asthma treatment. Pediatrics. 2012; 129:e1148. [PubMed: 22492763]

13. National Center for Complementary and Alternative Medicine (NCCAM). What is CAM? [Web Site]. 2007. [cited 2007 March 8]; Available from: http://nccam.nih.gov/health/whatiscam/

14. Slader CA, et al. Complementary and alternative medicine use in asthma: Who is using what? Respirology. 2006; 11:373-387. [PubMed: 16771907]

15. Braganza S, Ozuah PO, Sharif I. The use of complementary therapies in inner-city asthmatic children. Journal of Asthma. 2003; 40(7):823-7. [PubMed: 14626339]

16. Pachter LM, Weller SC. Acculturation and compliance with medical therapy. Journal of Developmental and Behavioral Pediatrics. 1993; 14(3):163-168. [PubMed: 8340470]

17. Pachter LM, Cloutier MM, Bernstein BA. Ethnomedical (folk) remedies for childhood asthma in a mainland Puerto Rican community. Archives of Pediatrics and Adolescent Medicine. 1995; 149:982-988. [PubMed: 7655603]

18. Conn K, et al. The impact of parents' medication beliefs on asthma management. Pediatrics. 2007; 120(3):e521-e526. [PubMed: 17766496]

19. Koster ES, et al. Uncontrolled asthma at age 8: The importance of parental perception toward medication. Pediatric Allergy and Immunology. 2011; 22:461-468.

20. McQuaid EL, et al. Beliefs and barriers to medication use in parents of Latino children with asthma. Pediatric Pulmonology. 2009; 44:892-898. [PubMed: 19672958] 
21. Flores G, et al. Access barriers to health care for Latino children. Archives of Pediatric and Adolescent Medicine. 1998; 152:1119-1125.

22. Shen J, Oraka E. Complementary and alternative medicine (CAM) use among children with current asthma. Preventive Medicine. 2012; 54:27-31. [PubMed: 22015560]

23. Adams SK, Murdock KK, McQuaid EL. Complementary and alternative medication (CAM) use and asthma outcomes in children: an urban perspective. Journal of Asthma. 2007; 44:775-782. [PubMed: 17994410]

24. Canino G, et al. Issues and methods in disparities research: The Rhode Island-Puerto Rico asthma center. Pediatric Pulmonology. 2009; 44(9):899-908. [PubMed: 19658111]

25. Esteban CA, et al. Conundrums in childhood asthma severity, control, and health care use: Puerto Rico versus Rhode Island. Journal of Allergy and Clinical Immunology. 2009; 124(2):238-244.e5. [PubMed: 19615729]

26. Fritz G, et al. Ethnic differences in perception of lung function: A factor in pediatric asthma disparities? American Journal of Respiratory and Critical Care Medicine. 2010; 182:12-18. [PubMed: 20299534]

27. McQuaid EL, et al. Pediatric asthma management in the family context: The family asthma management system scale. Journal of Pediatric Psychology. 2005; 30:492-502. [PubMed: 16055487]

28. Global Initiative for Asthma. Revision: GINA Report, Global strategy for asthma management and prevention. Global Strategy for Asthma Management and Prevention. 2006

29. Horne R, Weinman R. Patients' beliefs about prescribed medicines and their role in adherence to treatment in chronic physical illness. Journal of Psychosomatic Research. 1999; 47(6):555-567. [PubMed: 10661603]

30. Koinis-Mitchell D, et al. Latino caregivers' beliefs about asthma: causes, symptoms, and practices. Journal of Asthma. 2008; 45(3):205-210. [PubMed: 18415827]

31. McQuaid EL, et al. Medication adherence in pediatric asthma: Reasoning, responsibility, and behavior. Journal of Pediatric Psychology. 2003; 28(5):323-333. [PubMed: 12808009]

32. Celano MP, et al. Treatment adherence among low-income, African American children with persistent asthma. Journal of Asthma. 2010; 47(3):317-322. [PubMed: 20394517]

33. McNally KA, et al. Adherence to combined montelukast and fluticasone treatment in economically disadvantaged African American youth with asthma. Journal of Asthma. 2009; (46):921-927. [PubMed: 19905919]

34. Lasmar L, et al. Adherence rate to inhaled corticosteroids and their impact on asthma control. Allergy. 2009; 64(5):784-9. [PubMed: 19183166]

35. Barnes, PM., et al. Complementary and alternative medicine use among adults: United States, 2002. U.S. Department of Health and Human Services, Centers for Disease Control; 2004.

36. Partridge MR. In what way may race, ethnicity or culture influence asthma outcomes? Thorax - An International Journal of Respiratory Medicine. 2000; 55(3)

37. Bailey E, et al. Culture-specific programs for children and adults from minority groups who have asthma. Cochrane Database of Systematic Reviews. 2009; (1):Art. No.: CD006580.10.1002/14651858.CD006580.pub3

38. Pachter, L. Working with Patients' Health Beliefs and Behaviors: The Awareness-AssessmentNegotiation Model in Clinical Care. Ross Products Division, Abbott Laboratories; Columbus, Ohio: 2000. 


\section{What's New}

This study evaluates associations between CAM use and parental beliefs about conventional medications and barriers to obtaining these medications among Latino and non-Latino white (NLW) families of children with asthma, and how this use relates to medication adherence. CAM use was positively related to adherence in NLWs, and unrelated among Latinos. 


\section{Total Sample \\ $\mathrm{n}=805$}

\section{Persistent Asthma Only \\ $\mathrm{n}=609$}

\section{CAM Data \\ $\mathrm{n}=574$}

\section{Medication Adherence Subset $\mathrm{n}=259$}

Figure 1.

Participant Flow 


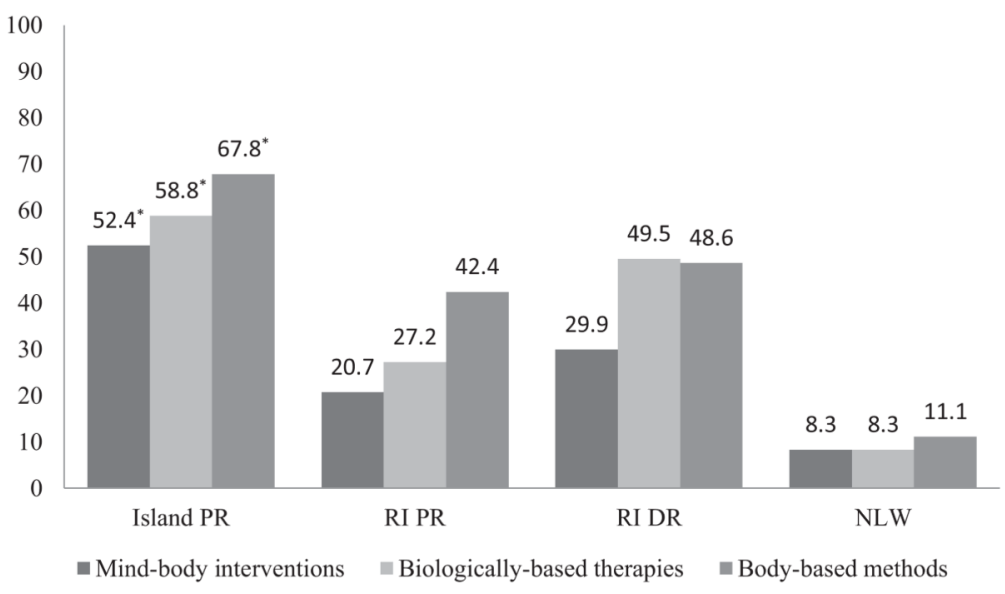

Figure 2. Percentages of CAM Type by Site/Ethnicity

Note $. \mathrm{PR}=$ Puerto Rico; DR = Dominican Republic; NLW = Non-Latino White. ${ }^{*} p<.001$ from Chi-square analysis comparing CAM type by site/ethnicity. 
Table 1

Demographics of children and caregivers

\begin{tabular}{|c|c|c|c|c|}
\hline & Total Sample $(n=574)$ & $\begin{array}{l}\text { No Adherence Data } \\
\text { Subsample }(n=315)\end{array}$ & $\begin{array}{c}\text { Adherence Data } \\
\text { Subsample }(n=259)\end{array}$ & $p$ \\
\hline Child ethnicity/site, $n(\%)$ & & & & $<.001$ \\
\hline Island PR & $267(46.5 \%)$ & $190(60.3 \%)$ & $77(29.7 \%)$ & \\
\hline RI PR & $92(16 \%)$ & $41(13.0 \%)$ & $51(19.7 \%)$ & \\
\hline RI DR & $107(18.6 \%)$ & $51(16.2 \%)$ & $56(21.6 \%)$ & \\
\hline RI NLW & $108(18.8)$ & $33(10.5 \%)$ & $75(29.0 \%)$ & \\
\hline Child age, $M(S D)$ & $10.54(2.48)$ & $10.73(2.51)$ & $10.32(2.43)$ & .053 \\
\hline Asthma severity, $n(\%)$ & & & & $<.001$ \\
\hline Mild persistent & $209(36.4 \%)$ & $132(41.9 \%)$ & $77(29.7 \%)$ & \\
\hline Moderate persistent & $231(40.2 \%)$ & $143(45.4 \%)$ & $88(34.0 \%)$ & \\
\hline Severe persistent & $134(23.3 \%)$ & $40(12.7 \%)$ & $94(36.3 \%)$ & \\
\hline Child gender, female, $n(\%)$ & $242(42.2 \%)$ & $133(42.2 \%)$ & $109(42.1 \%)$ & .974 \\
\hline Caregiver identity, $n(\%)$ & & & & .051 \\
\hline Biological mother & $545(94.9 \%)$ & $299(94.9 \%)$ & $246(95.0 \%)$ & \\
\hline Biological father & $12(2.1 \%)$ & $3(1.0 \%)$ & $9(3.5 \%)$ & \\
\hline Other female relative & $16(2.7 \%)$ & $12(3.8 \%)$ & $4(1.6 \%)$ & \\
\hline Other male relative & $1(.2 \%)$ & $1(.2 \%)$ & $0(0 \%)$ & \\
\hline Below poverty threshold, $n(\%)$ & $303(52.8 \%)$ & $59.4 \%$ & $116(44.8 \%)$ & $<.001$ \\
\hline $\begin{array}{l}\text { Combined caregiver years of education, } M \\
(S D)\end{array}$ & $12.72(2.69)$ & $12.59(2.67)$ & $12.88(2.71)$ & .118 \\
\hline Spanish language preference, $n(\%)$ & & & & .617 \\
\hline RI PR & $52(56.5)$ & $20(6.3 \%)$ & $32(12.4 \%)$ & \\
\hline RI DR & $92(86.0)$ & $45(14.3 \%)$ & $47(18.1 \%)$ & \\
\hline \multicolumn{5}{|l|}{ Years caregiver lived outside US, $M(S D)$} \\
\hline RI PR & $13.58(11.74)$ & $12.31(12.41)$ & $14.63(11.18)$ & .352 \\
\hline RI DR & $21.51(10.25)$ & $21.78(9.72)$ & $21.26(10.80)$ & .795 \\
\hline
\end{tabular}

Note. $\mathrm{PR}=$ Puerto Rico; DR = Dominican Republic; NLW = Non-Latino White. Comparisons between adherence subsamples made using chisquare or $t$ tests. 


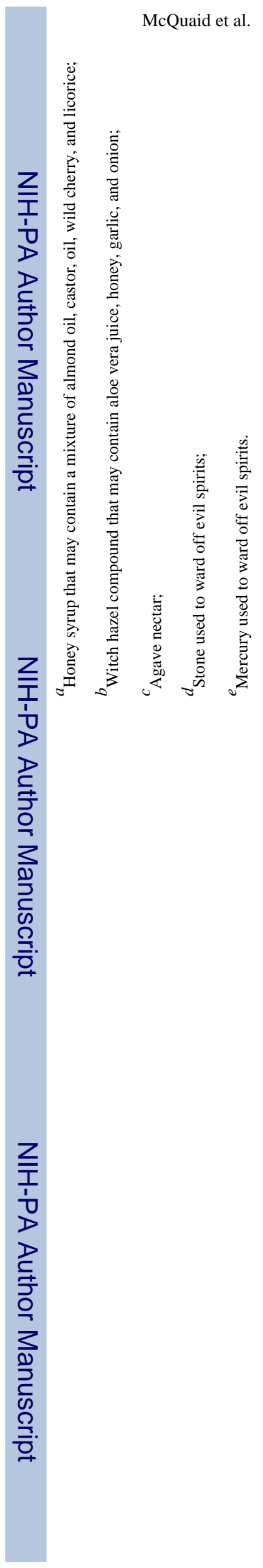

Acad Pediatr. Author manuscript; available in PMC 2015 March 01. 


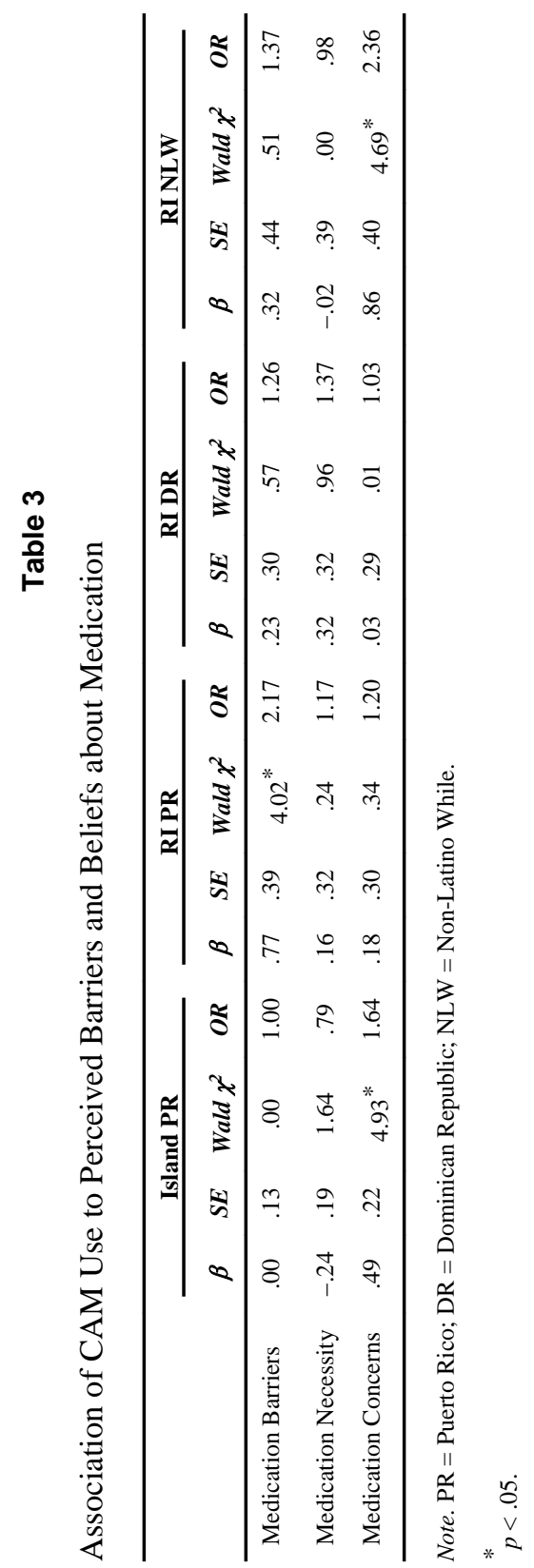




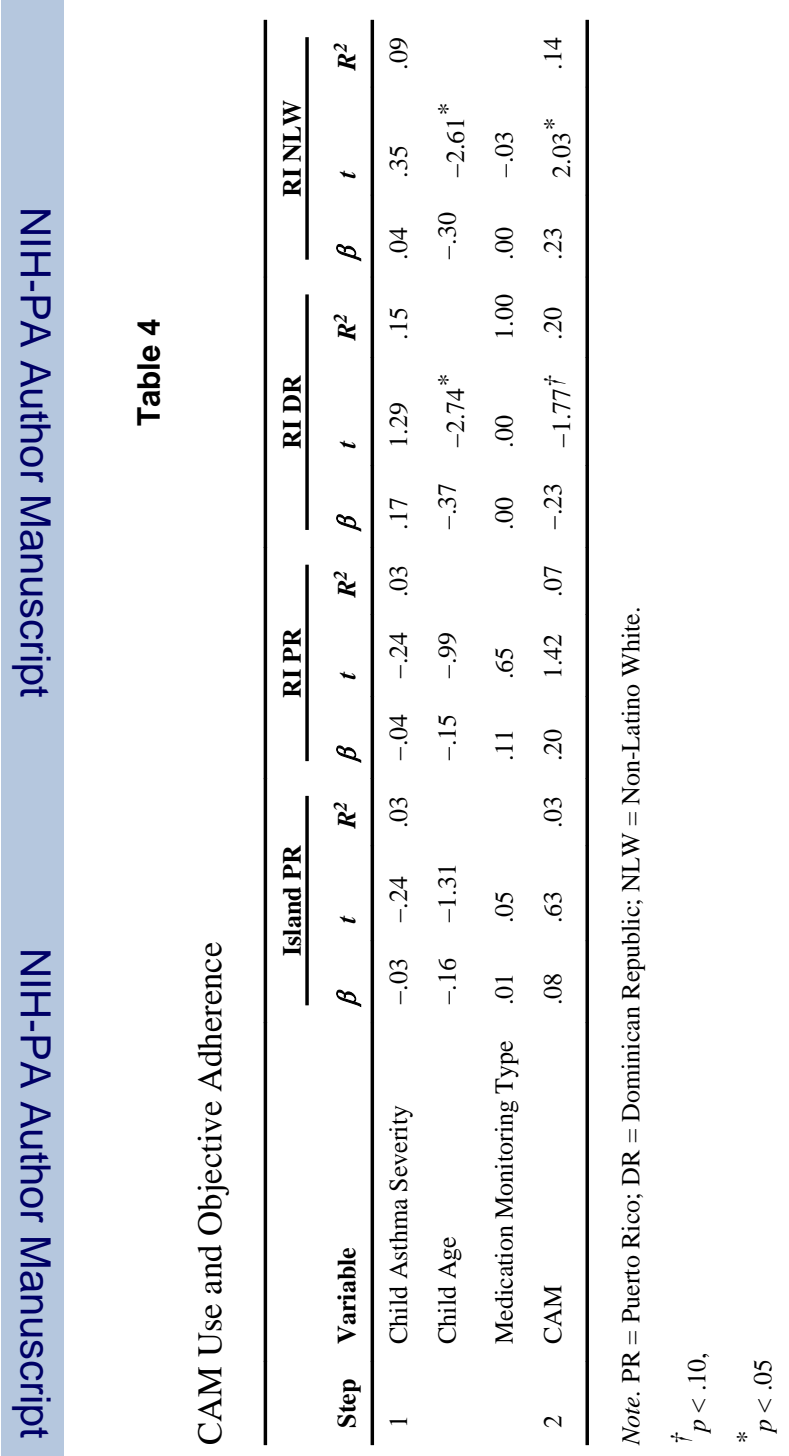

